\title{
A Case of Chronic Mesenteric Ischaemia Imitating Crohn's Disease
}

\author{
Maria Paparoupa, Frank Schuppert, Huy Ho \\ Department of Gastroenterology, Endocrinology and Diabetology, Klinikum Kassel, \\ University Hospital of Southampton, Kassel, Germany
}

Received: $29 / 05 / 2015$

Accepted: 16/06/2015

Published: $16 / 07 / 2015$

How to cite this article: Paparoupa M, Schuppert F, Ho H. A case of chronic mesenteric ischaemia imitating crohn's disease. EJCRIM 2015;2:doi: $10.12890 / 2015$

Conflicts of Interests: The authors declare that they have no conflicts of interest in this research.

Aknowledgements: The authors declare that they have the Ethics' Committee authorization to publish this case.

This article is licensed under a Commons Attribution Non-Commercial 4.0 License

\begin{abstract}
Objectives: we present an atypical case of chronic mesenteric ischaemia with weight loss as the only clinical manifestation and endoscopic findings imitating Crohn's disease.

Materials and methods: CT angiography of the abdomen confirmed the diagnosis of mesenteric ischaemia after total occlusion of the coeliac trunk and superior mesenteric artery.

Results: The patient died due to severe sepsis as a result of extended bowel infarction.

Conclusions: The diagnosis of chronic mesenteric ischaemia requires a high degree of clinical suspicion and can be life-saving if made quickly.
\end{abstract}

\section{LEARNING POINTS}

- Unintended weight loss can be a clinical manifestation of chronic mesenteric ischaemia.

- Post-prandial abdominal pain is not always present especially if it is caused by non-occlusive visceral stenosis.

- Endoscopic findings can imitate chronic inflammatory disease due to preserved moderate bowel tissue perfusion.

\section{KEYWORDS}

Chronic mesenteric ischemia, weight loss, Crohn's disease, video capsule endoscopy.

\section{INTRODUCTION.}

Ischaemic bowel disease is classified as colonic or mesenteric ischaemia and can have an acute or chronic clinical course. However, chronic mesenteric ischaemia can be easily overlooked as collateral vascular bands ensure moderate but sufficient bowel tissue perfusion and the patient's symptomatology remains vague until acute arterial occlusion occurs ${ }^{[1]}$.

\section{CASE REPORT}

A 51-year-old German woman presented to our gastroenterology department due to unintended weight loss of $13 \mathrm{~kg}$ in the previous 12 months and persistent low back pain for several years. No abdominal pain, signs of gastrointestinal bleeding, fever or diarrhoea was reported. The patient had been already referred to the orthopaedic clinic and was receiving high doses of opiates, without any relief of her symptoms. She had a former medical history of asthma and depression. Of note, her brother had died several months earlier. Life-long cigarette consumption was estimated to be 40 pack-years and a moderate alcohol intake was reported.

Physical examination revealed diffuse tender palpation of her abdomen and local tenderness of the lower spinal column. There was no peritonism and intestinal peristalsis was normal. The patient had a body mass index (BMI) of 15 (43 kg, $168 \mathrm{~cm})$. Laboratory tests revealed malnutrition with hypoalbuminaemia of $2.38 \mathrm{~g} / \mathrm{dl}$ (reference: $4.02-4.76 \mathrm{~g} / \mathrm{dl}$ ) and elevated faecal calprotectin of $498 \mu \mathrm{g} / \mathrm{g}$ (reference: $10-31$ $\mu \mathrm{g} / \mathrm{g})$. Upper gastrointestinal endoscopy detected three ventricular ulcers, while flexible colonoscopy was completed without pathological findings. A contrast-enhanced abdominal and pelvic CT scan shown bilateral adenomas of the adrenal glands and a $7.5 \mathrm{~cm}$ uterine leiomyoma with a central necrotic region. Multiple small bowel loops had a thickened intestinal wall, compatible with enteritis of unclear origin. In order to further investigate the small bowel wall, we decided to perform a video capsule endoscopy. Multiple superficial erosions and aphthous ulcerations were seen, findings which were compatible with Crohn's disease (Figures. 1 and 2).

The patient was discharged with the diagnosis of chronic pain syndrome due to underlying depression and previous drug overdose with a potential eating disorder. The suspected diagnosis of Crohn's disease (elevated faecal calprotectin and capsule endoscopy findings) was not histologically confirmed, as the biopsies of ventricular ulcers revealed no signs of inflammatory infiltration. However, the patient was 
treated empirically with corticosteroids and referred to our obstetrics and gynaecology colleagues for a complete hysterectomy due to the necrotic uterine leiomyoma.

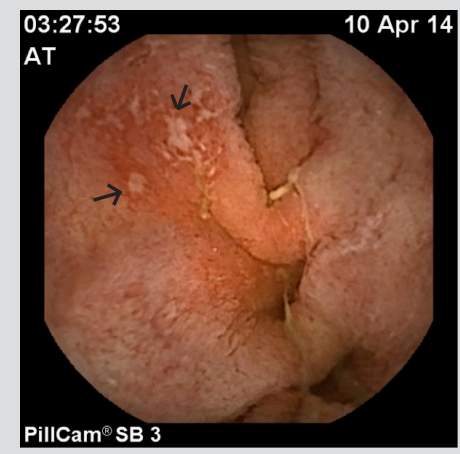

Figure 1: Video capsule endoscopy (PillCam SB3) showing multiple superficial erosions and aphthous ulcerations in the jejunum compatible with Crohn's disease findings.

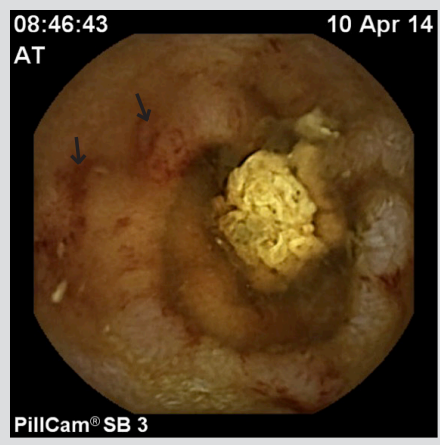

Figure 2: Video capsule endoscopy (PillCam SB3) showing multiple superficial erosions in the ileus.

During her second hospitalization in the gynaecology department and several days after she had undergone a laparoscopic procedure for the uterine leiomyoma, the patient developed clinical and laboratory signs of systemic inflammatory response syndrome (SIRS). A conventional abdominal x-ray demonstrated a massive small intestine ileus and a consequent CT angiography of the abdomen and pelvis showed total occlusion of the coeliac trunk and superior mesenteric artery (Figures 3 and 4). A diagnostic laparotomy confirmed the diagnosis of advanced bowel necrosis following acute mesenteric infarction and the patient died several hours later from severe sepsis.

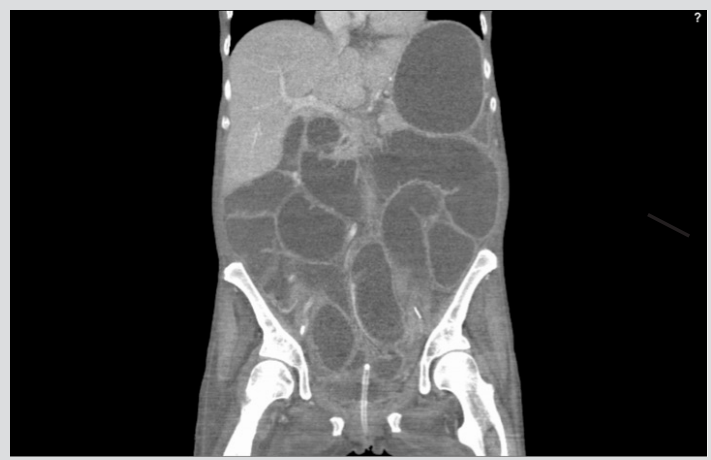

Figure 3: CT scan of the abdomen showing massive small intestine ileus.

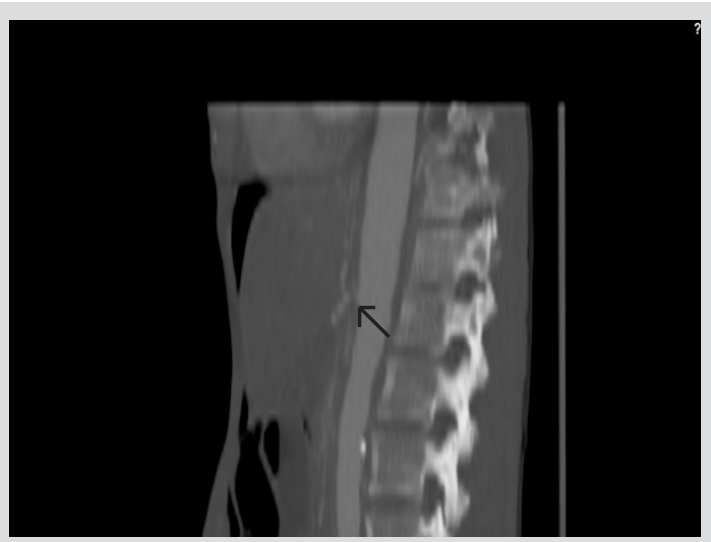

Figure 4: Abdominal CT angiography demonstrating total occlusion of the coeliac trunk and superior mesenteric artery directly after

they arise from the aorta.

\section{DISCUSSION}

In our case, unmistakable weight loss and malnutrition were the main manifestations of chronic mesenteric ischaemia. There are sufficient data demonstrating that unintentional weight loss can be caused by chronic reduced splanchnic blood flow ${ }^{[2]}$. It is remarkable that typical symptoms, such as post-prandial abdominal pain and stool irregularities, were not present in our case. Intestinal angina occurs when the genuine and collateral vessels can no longer compensate for the increased post-prandial demand by the gastrointestinal tract and liver ${ }^{[3]}$.

The persistent low back pain unfortunately confused the clinical evaluation of this case, leading to the diagnosis of chronic pain syndrome due to underlying depression and a potential eating disorder. Early radiological signs of moderate bowel tissue perfusion such as thickening of the intestinal wall and endoscopic findings of superficial ulcerations were evaluated as enteritis of unclear origin, most likely due to Crohn's disease. The elevated marker of faecal calprotectin supported our diagnostic hypothesis, although no histological confirmation was obtained. Subsequent radiological signs of mesenteric ischaemia can be bowel wall thickening, pneumatosis and peritoneal fluid ${ }^{[4]}$. The absence of endoscopic findings in the colon demonstrates the presence of a partial segmental occlusion of a mesenteric branch ${ }^{[5]}$.

In our case, atherosclerotic vascular stenosis was the most likely underlying pathology causing the low visceral blood flow. Review of the first CT scan of the abdomen showed $70 \%$ stenosis was present. This was initially underestimated and the final diagnosis was made after total arterial occlusion with subsequent infarction, paralytic ileus and SIRS. Atherosclerotic disease of the mesenteric vessels should be considered in any life-long smoker even at younger ages ${ }^{[6]}$. 


\section{CONCLUSIONS}

Unintended weight loss can be a manifestation of chronic mesenteric ischaemia, even if abdominal pain is absent. A thickened intestinal wall or endoscopic findings of superficial ulcerations can be early signs of moderate bowel tissue perfusion. The diagnosis of chronic mesenteric ischaemia requires a high degree of clinical suspicion and can be life-saving if made early.

\section{REFERENCES}

1. Zacho HD, Abrahamsen J. Chronic intestinal ischaemia: diagnosis. Clin Physiol Funct Imaging 2008;28:71-75.

Clifton WL, Kneitz A, Cohn WE, Delgado RM 3rd. Weight loss caused by visceral artery disease. Tex Heart Inst J 2013;40:320-322.

Kilsby A, Pasha Y. Chronic mesenteric ischaemia: a battery of negative tests in a patient with episodic abdominal pain, weight loss and diarrhoea. BMJ Case Rep 2013 Jan 2;2013.

4. Hohenwalter EJ. Chronic mesenteric ischemia: diagnosis and treatment. Semin Intervent Radiol 2009;26:345-351.

4. Hohenwalter EJ. Chronic mesenteric ischemia: diagnosis and treatment. Semin Intervent Radiol 2009;26:345-351.
5. Van De Winkel N, Cheragwandi A, Nieboer K, van Tussenbroek F, De Vogelaere K, Delvaux G. Superior mesenteric arterial branch occlusion causing partial jejunal ischemia: a case report. J Med Case Rep 2012;6:48.

6. Al-Hammash SM, Al-Ethawi Ael-S, Ismail KA. Endovascular treatment of chronic mesenteric ischemia in an adolescent male: case report. F1000Res 2013;2:277. 